Proc. Indian Acad. Sci. (Earth Planet, Scl,), Vol, 90, Nựmber 2, July 1981, pp. 147-153

- Printed in India.

\title{
Determination of partial geoidal parameters over the Indian sub-continent
}

\author{
V K GAUR \\ Department of Earth Sciences, University of Roorkee, Roorkee 247672, India
}

MS received 12 November 1979; revised 20 March 1981

\begin{abstract}
The paper presents values of partial geoidal parameters $N, \xi$ and $\eta$ which define the departure of the geoid from the reference spheroid, at $1^{\circ}$ intervals over the Indian subcontinent. These values represent contributions arising from the gravity anomaly data over the entire earth's surface, except for that from a $6^{\circ} \times 6^{\circ}$ element around the immediate neighbourhood of the point. Complete values of these parameters at a point can be obtained simply by adding to these partial values, contributions from the $6^{\circ} \times 6^{\circ}$ element circumscribing that point. The objective of the study was to provide a ready basis for updating the geoidal parameters at the initial reference point as and when the density and quality of local gravity data around it improve. These computations once made, would also facilitate calculation of geoidal parameters at a fairly large number of astro-geodetic stations apart from that at the initial reference point, which should lead to considerably more precise value of the absolute datum of the Indian geodetic dasystem.
\end{abstract}

Keywords. Geoidal parameters; Indian subcontinent.

\section{Introduction}

The figure of the earth is a level surface whose potential is principally* determined by the gravitational attraction of the earth's mass and the centrifugal force of its axial rotation. By convention, this level surface coincides with the mean sea level which very nearly gives a physical representation of an equipotential surface, and is called the geoid. The equipotential surface through a point, which naturally guides the instrument level, inevitably provides the reference at that point on the earth's surface for the determination of its astronomic coordinates. Owing to its irregular shape, however, the geoid cannot be used as a suitable basis for computing precise locations of widely separated points on the earth's surface. A possible alternative is to choose a perfect geometric reference surface closely approximating to the geoid and an appropriate system of geodetic coordinates. The standard choice is an ellipsoid of revolution or a spheroid of internationally agreed dimensions with its minor axis oriented parallel to the rotational axis of the earth. The departure of the geoid at any point of the earth's surface from the reference spheroid is

* A small effect due to the atmospheric shell amounts to a geoidal undulation of 2.3 metres 
usually defined by three geoidal parameters $N, \xi$ and $\eta$ where, $N^{* *}$ is the vertical distance between the geoid and the reference spheroid and, $\xi, \eta$ are respectively the N-S and E-W components of the angular difference between verticals to the two surfaces at the point in question. A given choice of these three parameters at the initial point, together with the adopted size and orientation of the reference spheroid, defines the datum of a geodetic system. Accordingly, there exist varied geodetic systems adopted in different parts of the world, some of which differ from one another quite markedly even though they are based on the same internationally agreed reference spheroid. Thus, whilst the Indian geodetic system, originally based on the Everest spheroid of 1830, was fitted to the International reference spheroid in 1927, its geoidal parameters were fixed only to suit local considerations. In consequence, the orientation of the Indian geodetic system and for that matter the position of the Indian geoid as a whole with respect to the reference spheroid, has uptil now remained arbitrary. These are, therefore, likely to differ appreciably from their absolute or true geocentric positions, the discrepancies increasing with the extent of the geodetic network. Geoidal parameters for points on the Indian subcontinent, have been computed based on the gravimetric method using global gravity data; and this exercise forms the subject matter of this paper.

Theoretically, one can compute the departure of the geoid from the reference spheroid using Stokes and Vening Meinesz integrals which require a complete knowledge of the earth's gravity field at every point of its surface. However, since the gravity field of the earth is not so regular as to be expressible in a mathematical form, the integrals must be evaluated numerically. The accuracy of computations will therefore depend on the size of the domains chosen for summation as well as on other factors related to the weighted functions and the input variances involved.

However, the near-linearity in the variations of the Stokes' function at large radial distances from a computation point and its progressively decreasing magnitude with distance, suggests that one could choose comparatively larger size area elements to divide the region beyond a certain distance from a point in question, without sacrificing the precision significantly. This is also true for the deflection components as the linearity of the Vening Meinesz function used in their computation is even more pronounced at larger distances.

\section{Computations of the partial geoidal parameters}

In order to carry out the computations numerically, each area element or mesh is assigned a value of the gravity anomaly at its centre, equal to the mean value of the gravity anomaly over the mesh. As explained above the, size of the meshes in the region of integration far from the point at which

\footnotetext{
** Neglecting the zero-order undulation of the geoid $N_{0}=\left[(K \delta M / 2 R \bar{\gamma})-(R / 2 \bar{\gamma}) \Delta g_{0}\right]$ on the assumption that both, $K \delta M$ which is the product of the gravitational constant and the mass deviation of the reference spheroid from that of the actual earth, and $\Delta g_{0}$ which is the mean gravity anomaly over the entire earth's surface are zero (vide Rapp and Rummal Report No. 233, Ohio State University).
} 
computations are made; could be chosen to be fairly large; but it has to be small in the region of integration immediately surrounding this point in order to gain comparable precision.

For computations of the geoidal parameter at points within the Indian-subcontinent, the total domain of integration outside the $6^{\circ} \times 6^{\circ}$ element in the immediate neighbourhood of a point was divided into (i) a far region made up of 5-degree equal area elements and (ii) a nearer one with finer size $1^{\circ} \times 1^{\circ}$ elements. Since the requisite accuracy of gravity data in the two regions is much less than that required in the immediate neighbourhood of a point, it was considered desirable to compute the partial geoidal parameters at $1^{\circ}$ intervals, as these could then form a ready basis for calculating the full values of these parameters at any point simply by adding to these the contributions arising from the immediate neighbourhood of that point. As the precision of the full value is primarily determined by the latter, these determinations could be revised as and when good quality gravity data around a desired point become available.

The partial geoidal parameters are based on the mean gravity values over 5degree square equal area elements, published by the Ohio State University (Rapp 1977) and that over the one-degree square elements supplied by the Defence Mapping Agency (NASA) and recently issued by the Survey of India, after being reduced to the GRS-67 System.

\section{Gravimetric method for determining geoidal parameters}

The gravimetric method for determining the absolute geoidal undulations $N$ and deflections of the vertical $\xi$ and $\eta$ at a point on the earth's surface is based on the Stokes and Vening Meinesz formulae which relate these to the surface integral of weighted mean gravity anomalies $\Delta g$ over the entire earth's surface as follows :

$$
\begin{aligned}
& N=\frac{1}{4 \pi \bar{\gamma} R} \iint_{S} \Delta g S(\psi) d s, \\
& \text { and }\left\{\begin{array}{c}
\xi \\
\eta
\end{array}\right\}=-\frac{\operatorname{cosec} 1^{\prime \prime}}{4 \pi \bar{\gamma} R^{2}} \iint_{s} \Delta g \frac{\partial S(\psi)}{\partial \psi}\left\{\begin{array}{l}
\cos \alpha \\
\sin \alpha
\end{array}\right\} d s,
\end{aligned}
$$

where, $d s=R^{2} . \sin \psi d \psi d \alpha$

$\boldsymbol{R}=$ mean radius of the earth

$\bar{\gamma}=$ mean normal gravity value over the spheroid

$\psi=$ angular distance of the variable element $d s$ from the computation point

$\alpha=$ azimuth of $d s$ at the computation point

$S(\psi)=$ the Stokes' function given by :

$$
S(\psi)=\operatorname{cosec} \frac{\psi}{2}+1-5 \cos \psi \log _{\theta}\left(\sin \frac{\psi}{2}+\sin ^{2} \frac{\psi}{2}\right)-6 \sin \frac{\psi}{2} .
$$


To evaluate the above integrals numerically the earth's surface is divided by a suitable grid system into area elements. The advantages and disadvantages of the meridian-parallel grid system, and another producing equal area elements are briefly outlined below.

\subsection{Meridian-parallel grid}

This grid is formed by meridian lines drawn at equal angular intervals and parallels of latitudes, also at the same interval. The area of an element formed by these grids will, however, vary according to its position owing to latitudelongitude asymmetry, being over 25 times larger in the equatorial region as compared with that in the polar region. For an angular separation of $5^{\circ}$, the total number of such area elements will be 2592 .

\subsection{Equal area grid}

Another way of dividing the earth's surface would be by a grid system formed by parallels of latitudes drawn at equal angular intervals, and longitudes whose angular separation is varied to ensure that the area of each element is the same. Using a $5^{\circ}$ angular separation between latitudes, this would mean that the angular separation between longitudes will vary from $5^{\circ}$ in the equatorial region to as much as $120^{\circ}$ in the polar region, the total number of elements thus formed, being 1654 (Ray 1978).

The meridian-parallel grid system should obviously be more suitable for computations for economizing the use of mean gravity anomalies as input data. The computation points in this case may be chosen to coincide with the grid corners for which the coefficients to be multiplied to $\Delta g$ would need to be evaluated only once in respect of the entire latitude belt and on only one side of the meridian, as has been done by Tanni (1948). Moreover, it constrains the minimum distance from a computation point to the centre of an element representing the mean gravity anomaly over it, to be equal to about 3.5 degrees, thereby making for uniform precision of computation at all points (Ray 1978).

However, for computation points coinciding with astrogeodetic stations such as the origin of a local geodetic system, the use of equal area grid would be more economical, the number of area elements being $35 \%$ less as compared with that for the meridian-parallel type. Moreover, the surface of each area element being nearly the same for all, the relative weightages are equal.

For the present work, equal area grids were formed and the updated Ohio State University data has been used. Moreover, geoidal parameters have been reduced to the GRS 67 system as the International Spheroid has been superseded by the now universally accepted GRS Spheroid.

\section{Inner limits of the far and near regions}

The effect of the far region, from a certain distance outwards right up to the antipodes, varies smoothly and is therefore, interpolable. Its inner limit can be 
calculated for a desired accuracy of determination. A distance of $20^{\circ}$ is a generally accepted recommendation for the computation of $\mathrm{N}$ and $15^{\circ}$ for those of $\xi$ and $\eta$. Accordingly, the inner boundaries of this region were fixed at around the 15 th degree of latitude and longitude from those circumscribing the Indian subcontinent.

The near region extended inwards from this boundary upto the $6^{\circ} \times 6^{\circ} \mathrm{im}$ mediate neighbourhood of the point at which partial geoidal parameters were calculated. This region was divided by a grid system, defining elements of size $1^{\circ} \times 1^{\circ}$ throughout.

Partial geoidal parameters at points $1^{\circ}$ apart over the Indian subcontinent, were then calculated by adding the contributions from the far and the near regions. Contributions to the integrals arising from the immediate neighbourhood of the origin of the Indian triangulation system at Kalianpur H. S. for which data of requisite precision was available, was also carried out. For other points, however, this exercise remains to be done in the future as and when adequate high quality data is obtained. But whenever that happens, computation of the full geoidal parameters will be reduced to the simple task of just computing these last contributions and adding the value so obtained to the partial geoidal values at the corresponding' point.

\section{R esults}

Results of computations for the partial geoidal parameters $N, \xi$ and $\eta$ are shown in figure 1. The three values written in various meshes represent the values of $N, \xi$ and $\eta$ in that order and refer to the left hand upper corner point of the mesh. As already mentioned to obtain the full values of these parameters at a point, it would only be necessary to compute the contribution at that point arising from the $6^{\circ} \times 6^{\circ}$ square in the immediate neighbourhood of the point and add this to the partial geoidal values, or values interpolated therefrom if the point does not coincide with the grid intersections. For example, the contribution arising from its immediate neighbourhood at Kalianpur $\mathbf{H}$. S. which is the origin of the Indian triangulation system, was computed as fortunately, adequate good quality and high density gravity data was available around this point. This contribution to $N, \xi$ and $\eta$ duly corrected for the atmospheric refraction effect amount to $-0 \cdot 8,+1^{\prime \prime} 0$ and $+2^{\prime \prime} \cdot 4$ respectively. As the partial geoidal parameters at this point obtained by linear interpolation happen to be equal to $-55.79 \mathrm{~m},-3 \cdot 05^{\prime \prime}$ and $+1^{\prime \prime} .68$ respectively, the full values can simply be obtained by adding them. Accordingly, we obtain for Kalianpur H. S., the following complete values of the geoidal parameters :

$$
\begin{aligned}
& N=-56.59 \mathrm{~m} \\
& \xi=-2.05^{\prime \prime} \\
& \eta=+4.07^{\prime \prime}
\end{aligned}
$$

Similar full values of the geoidal parameters, when computed at a number of astrogeodetic stations, will lead to a much more precise determinaton of the absolute datum of the Indian geodetic system. 


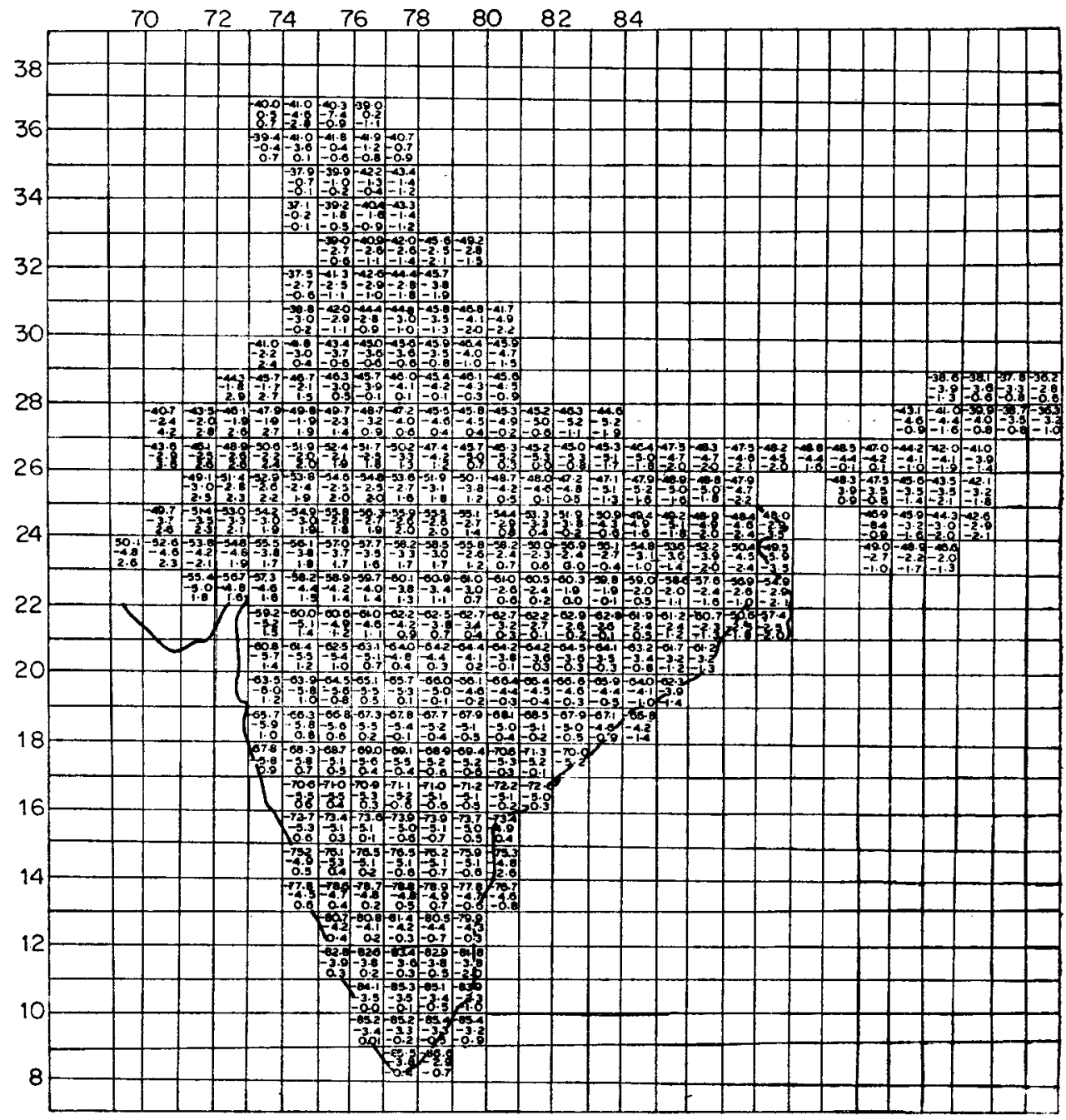

Figure 1. Map showing partial geoidal parameters $N, \xi$ and $\eta$ contributed by the gravity anomaly effects over the entire earth except for that arising from a $\left(6^{\circ} \times 6^{\circ}\right)$ square immediate neighbourhood of the point concerned. The three values entered in each mesh represent the values of $N, \xi$ and $\eta$ respectively in that order and refer to the left hand and upper corner of that mesh, excluding the correction due to the atmospheric shell of $-2.3 \mathrm{~m}$ to be applied to all $N$ values.

\section{Acknowledgements}

The author is indebted to Dr J C Bhattacharji for many stimulating discussions. The computer programs were developed by Dr P K Ray and the data obtained from Dr Rapp and the Survey of India, whose assistance is gratefully acknowledged. 


\section{References}

Bhattacharji J C 1979 GRS 67 Geoid and deflections in India (Manuscript)

Bhattacharji J C and Ray P K 1978 Proposed absolute datum for Indian Geodetic system, Presented at International Gravity commission held at Paris during Sept. 12-16 1978

Rapp R H 1977 Potential coefficient determination from $5^{\circ}$ terrestrial gravity data Report No. 251 Dept. Geodetic Science

Rapp R H and Rummel R Report No. 233, Ohio State University

Ray P K 1978 Absolute orientation of Indian geodetic system by gravimetric method Ph. D. Thesis, University of Roorkee

Tanni L 1948 On the continental undulations of the geoid as determined from the present gravity material Inst. Int. Assoc. Geode, Helsinki, No. 18 